\title{
On an MC-CDMA System Operating with Distinctive Scenarios of Antenna
}

\author{
Joy Iong-Zong CHEN, Hau-Wei HUANG, Kai Zhi ZHUANG \\ Department of Communication Engineering, Dayeh University, Dacun Changhua, Taiwan, China \\ Email:jchen@mail.dyu.edu.tw \\ Received June 10, 2009; revised July 12, 2009; accepted July 20, 2009
}

\begin{abstract}
In this paper the impact of distinctive structure of antenna with branch correlation for an OFDM (orthogonal frequency division multiplexing)-based system, MC-CDMA (multi-carrier coded-division multiple-access) system, operating over the frequency selective fading environments is studied. For the reason of accordance with the working environments in the real world applications (urban areas) the correlated-Nakagami- $m$ fading is adopted. Furthermore, the performance evaluation with average BER (bit error rate) formulas of MC-CDMA system with MRC (maximal ratio combining) diversity was derived with an alternative method of the complementary error function. The illustrated results are not only discussing the effect that comes from triangular, linear, and circular antenna array constructions, but the factors of branch correlation are also analyzed. Generally, it is known that the more the received branch number is, the more superior system performance of a multiple-access system will become. It is interesting to contrast to the geometric of the antenna array, that is, the little shape changing of the antenna is, the worse inferior system performance arrive at.
\end{abstract}

Keywords: Circular Antenna Array, Linear Antenna Array, MC-CDMA Systems, MRC Diversity, Nakagami- $m$ Fading, Triangular Antenna Array

\section{Introduction}

For the purpose of overcoming ISI (inter-symbol interference) effect and reducing the channel fading in a transmission channel, multi-carrier modulation scheme has been adopted for high speed transmission applications. A number of multi-carrier modulation techniques have been proposed during the pass decade [1]. To support a wide area of services and high data rate by using a variety of techniques capable of achieving the highest possible spectrum efficiency is the main objective for future generations of wideband wireless communication systems. The CDMA (coded-division multiple-access) scheme has been applied as an attractive multiple access technology in both $2 \mathrm{G}$ (second-generation) and $3 \mathrm{G}$ (third- generation) wireless radio systems. In general, the multicarrier DS systems have already been proposed and can be categorized into two types: a parallel transmission-scheme of narrowband DS waveforms in the fre- quency domain, and a combination of OFDM (orthogonal frequency division multiplexing) and CDMA [1]. The available frequency spectrum of carrier wave is divided into $M$ equal band of subcarriers in the former systems. These subcarriers are used to carry a narrowband DS waveform and the number of subcarriers is usually much less than the processing gain. In the latter system, each chip modulates a different carrier conveying a narrowband waveform rather than a DS waveform, and the number of carriers should be equal to the processing gain.

On the other hand, due to the advantages of spectrum efficient, interference immune, high date rate, and insensitivity to frequency selective channel, etc. Such that multiple access system bases on direct sequence CDMA (coded-division multiple-access) have drawn recently interest in the application of wireless radio systems [1]. Especially, multi-carrier CDMA (MC-CDMA) appears to be a considerable candidate for future mobile radio 
communication system. The MC-CDMA system based on the spread spectrum techniques. There are a lot of previous researches have been published for investigation about the issues of MC-CDMA system. Besides, the BER (bit error rate) analysis of MC-CDMA based on considering different kinds of assumptions, so far, have been dedicated in numerous previously researches [1-3]. In [2] the authors analyzed the BER (bit error rate) performance of uplink MC-CDMA system over frequency selective Nakagami- $m$ fading with MRC (maximal ratio combining) and EGC receptions. The performance evaluation of MC-CDMA over multipath fading channels was studied in [3]. The results presented in [4] are for uplink channel using MRC with the assumed frequency offsets condition in correlated fading. The performance of MC-CDMA in non-independent Rayleigh fading was studied in [5]. In [6], which by use of the method of CF (characteristic function) and residue theorem to calculate the performance for downlink MC-CDMA system. Both of the envelopes and phases correlation are considered in [7] to evaluate the performance of a MC-CDMA system operates in Rayleigh fading channel. The literature in [8] illustrated the error probability for MC-CDMA systems assumed that the transmission channel is in Nakagami- $m$ fading, and the postdetection of EGC (equal gain combining) is considered.

The different construction, linear, triangular, and circular antenna arrays are deployed as the environments for evaluating the system performance of uplink MC-CDMA systems in this paper. Moreover several expressions of BER performance are derived not only for the previously mentioned scenarios, but with also the assumption of correlated fading channels, which is assumed characterized as correlated-Nakagami- $m$ statistics. An average BER formula closed-form is obtained via the pdf (probability density function) of Gamma variates distribution to avoid the difficulty of explicitly obtaining the pdf for the SNR (signal-to-noise ratio) at the MRC output. The results from this paper partly analyze and show how the channel correlation affects the system performance of MC-CDMA systems, and explore the phenomena of different incident angular of waveform into an antenna array. The rest of this paper is organized as follows: Section 2 gives a description of the MC-CDMA system model, the correlated-Nakagami- $m$ fading channel model, and the receiver model of MC-CDMA system. The performance of MC-CDMA operating in uncorrelated and correlated fading cannel is carried out in Section 3. There are numerically results shown in Section 4. Finally, Section 5 draws briefly conclusions.

\section{MC-CDMA System Models}

\subsection{Transmitter Model}

An uplink MC-CDMA system model is considered for the study. Assuming that exist $K$ simultaneous users are with $N$ subcarriers within a signal cell. Any effect of correlation among users is going to be ignored by assuming the number of users is uniformed of distribution. As shown in Figure 1, a signal data symbol is replicated into $N$ parallel copies. The signature sequence chip with a spreading code of length $L$ is used to BPSK (binary phase shift keying) modulated each of the $N$ subscriers of the $k$-th user. Where the subcarrier has frequency $F / T_{b}$ $\mathrm{Hz}$, and where $F$ is an integer number $[1,3]$. The technical described above is same as to the performance of OFDM (orthogonal frequency division multiplexing) on a direct sequence spread-spectrum signal when set $F=1$. The larger values of $F$, the more transmit bandwidth increase. The transmitted signal the resulting transmitted baseband signal $S_{k}(t)$ corresponding to the $M$ data bit size can be expressed as

$$
S_{k}(t)=\sqrt{\frac{2 P}{N}} \sum_{m=0}^{M-1} \sum_{n=0}^{L-1} a_{k}[n] b_{k}[m] P_{T}(t) \operatorname{Re}\left[e^{j \omega_{n} t}\right]
$$

where $a_{k}[n] \in\{-1,1\}, \quad b_{k}[m] \in\{-1,1\}, \quad$ the sequencer $a_{k}[0], \ldots, a_{k}[L-1]$ and $b_{k}[0], \ldots, b_{k}[M-1]$ represent the signature sequence and the data bit of the $k$-th user, respectively, $P$ is the power of data bit, $M$ denotes the number of data bit, $N$ denotes the number of subcarriers, the $P_{T}(t)$ is defined as an unit amplitude pulse that is non-zero in the interval of $\left[0, T_{b}\right]$, and $\operatorname{Re}[\cdot]$ denotes the real part of a complex number, $\omega_{n}=2 \pi\left(f_{c}+n F / T_{b}\right)$ is the angular frequency of the $n$-th subcarrier.

\subsection{Channel Model}

A frequency-selective channel with $1 / T_{b} \ll B W_{c} \ll F / T_{b}$ is addressed in this paper, where $B W_{c}$ is the coherence bandwidth. This channel model means that each modulated subcarrier does not experience significant dispersion and with transmission bandwidth of $1 / T_{b}$, i.e. $T_{b} \gg T_{d}$, where $1 / T_{d}$ is the Doppler shift typically in the range of $0.3 \sim 6.1 \mathrm{~Hz}$ [1] in the indoor environment, and the amplitude and phase remain constant even the symbol duration $T_{b}$. In addition to, the channel of interest has the transfer function of the continuous-time fading channel assumed for the $k$-th user can be represented as

$$
H_{k}\left[f_{c}+i \frac{F}{T_{b}}\right]=\beta_{k, i} e^{j \theta_{k, i}}
$$


where $\beta_{k, i}$ and $\theta_{k, i}$ are the random amplitude and phase of the channel of the $k$-th user at frequency $f_{c}+i\left(F / T_{b}\right)$. In order to follow the real world case, the random amplitude, $\beta_{k, i}$ are assumed to be a set of $N$ correlated not necessarily identically distributed in one of our scenarios.

The equal fading severities are considered for all of the channels, namely, $m_{\ell}=m, \ell=1, \ldots, L$. The pdf of the fading amplitude for the $k$-th user with $i$-th channel, $\beta_{k, \ell}$, are assumed as r. v. (random variable) with the Nakagami- $m$ distribution, and given as [10]

$$
P(\beta)=\frac{2 \beta^{2 m-1}}{\Gamma(m)} \cdot\left(\frac{m}{\Omega}\right)^{m} \cdot \exp \left(-\frac{m \beta^{2}}{\Omega}\right), \quad \beta \geq 0
$$

where $\Gamma(\cdot)$ is the gamma function defined by $\Gamma(x)=\int_{0}^{\infty} t^{x-1} e^{-t} d t, \Omega=E\left[\beta^{2}\right]$ denoting expectation, the parameter $m$ of the amplitude distribution characterizes the severity of the fading, and it is defined as

$$
m=\frac{\Omega^{2}}{E\left[\left(\beta^{2}-\Omega\right)^{2}\right]} \geq 0.5
$$

It is well known that $m=0.5$ (one-sided Gaussian fading) corresponds to worst case fading condition, $m=1$ and $m=\infty$ correspond to Rayleigh fading (purely diffusive scattering) and the non-fading condition, respectively. As what follows, we consider these two cases.

First, with the assumption the propagation channels are assumed as i.i.d (identically independent distributed), then by using of the variable changing, the variable $\gamma$ is assigned as the fading intensity of the correlated channel, and let $\gamma=\beta^{2}$, then the pdf of $\gamma$ is given easily following as a Gamma distribution, which can be obtained by the processing of random stochastic as

$$
p_{r}(x, \ell, \gamma)=\frac{1}{\lambda_{\ell} \Gamma(\gamma)}\left(\frac{x}{\lambda_{\ell}}\right)^{\gamma-1} \exp \left(-\frac{x}{\lambda_{\ell}}\right)
$$

Let $\left[\gamma_{\ell}\right], \ell=0, \ldots, L-1$ be a set of $N$ correlated identically distributed, and all the figure parameters and the average power are assumed equivalent, that is, $\quad m_{i}=m_{j}=m$, and $\Omega_{i}=\Omega_{j}=\Omega$, where $i \neq j$, for $i, j=0, \ldots, L-1$. The power at the output of the MRC is a function of the sum of the squares of signal strengths, and is given as

$$
R=\sum_{\ell=0}^{L-1} \gamma_{\ell}
$$

\subsection{MC-CDMA Receiver Model}

A slowly varying fading channel is considered in this paper, that is, the channel parameters are unchanged over one bit duration $T_{b}$. For $K$ active transmitters, the received signal $r(t)$ can be written as

$$
\begin{aligned}
r(t) & =\sqrt{\frac{2 P}{N}} \sum_{k=0}^{K-1} \sum_{m=0}^{M-1} \sum_{n=0}^{L-1} \beta_{m, n} a_{k}[n] b_{k}[m] \\
& \times P_{T}\left(t-m T_{b}-\tau_{k}\right) \cos \left(\omega_{n} t+\theta_{m, n}\right)+n(t)
\end{aligned}
$$

where $n(t)$ is the AWGN (additive white Gaussian noise) with a double-sided power spectral density of $N_{0} / 2$. We can evaluate, the local-mean power, $P_{k, n}$, which is given as

$$
P_{k, n}=E\left[\beta_{k, n}^{2}\right] \frac{\rho}{N}
$$

where the total-mean power of the $k$-th user is defined to be $P_{k}=N \cdot P_{k, n}$, if the local-mean power of the subcarriers is assumed equal. Assuming that acquisition has been accomplished for the user of interesting $(k=0)$. In addition, the system operates synchronously with each user having the same clock is assumed, and the MRC diversity reception technique is considered in this paper. For the reason of using MRC, it is assumed that perfect phase correction can be obtained, i.e., $\hat{\theta}_{0, i}=\theta_{0 i}$. Demodulating each subcarrier includes applying a phase correction, $\hat{\theta}_{0, i}$, and a gain correction factor $d_{0, n}=\beta_{0, n} \cdot a_{0}[n]$ is multiplied by the $n$-th subcarrier signal as shown in Figure 2.

All the signals at the output of the correlators are combined with the MRC diversity scheme, and the results can be written as

$$
\gamma=\sum_{\ell=0}^{L-1} \gamma_{\ell}
$$

where $\gamma_{\ell}$ is the SNR at every branch. The branch number is assumed that equal to the subcarrier number, that is , $L=N$, in this paper. With all the assumptions for MRC combining, the decision variable $D_{0}$ of the $m$-th data bit reference user, and given by

$$
\begin{aligned}
D_{0}= & \frac{1}{T_{b}} \int_{m T_{b}}^{(m+1) T_{b}} r(t) \cdot \sum_{l=0}^{L-1} a_{0}[l] \cdot d_{0, l} \\
& \cdot \operatorname{Re}\left[e^{\left(w_{l} t+Q_{0,0}\right)}\right] d t \\
= & \mathrm{U}_{s}+I_{M A I}+\eta_{0}
\end{aligned}
$$

where $r(t)$ is the received signal shown in (10), $d_{0, i}$ is the gain factor for MRC diversity. The first term, $U_{S}$, in previous equation represents the desired signal, can be expressed as

$$
U_{S}=\sqrt{\frac{P}{2 N}} \sum_{\ell=0}^{L-1} \beta_{0, \ell}^{2} \cdot a_{0}[m]
$$


and the second term, $I_{M A I}$, is the MAI (multiple access interference) contributed from all other users which can be written as

$$
\begin{aligned}
I_{M A I}= & \sqrt{\frac{P}{2 N}} \cdot \sum_{k=1}^{K-1} \sum_{n=0}^{L-1} a_{k}[m] \cdot b_{k}[m] \cdot a_{0}[m] \\
& \cdot \beta_{k, m} \cdot \beta_{0, n} \cdot \cos \left(\theta_{k, n}^{\prime}\right)
\end{aligned}
$$

where $\theta_{k, n}^{\prime}=\theta_{0, n}-\theta_{k, n}$, where $\theta_{k, n}$ is i.i.d uniformly distributed over $[0,2 \pi)$, and the last term, $\eta_{0}$, in (13) is the AWGN.

\section{Performance Analysis}

A generalized average BER for the $k$-th user using coherent BPSK (binary phase shift keying) modulation scheme is derived in this section. For coherent demodulation in the presence of AWGN, the probability of error conditioned on the instantaneously $S N R$ can be expressed as [11]

$$
P_{e}(s)=0.5 Q(\sqrt{S N R})
$$

where the Gaussian Q-function is defined by

$$
Q(x)=\int_{x}^{\infty} 1 / 2 \pi e^{\left(-t^{2} / 2\right)} d t,
$$

and the received instantaneously $S N R$, which conditioned on $\gamma_{0, n}=\beta_{0, n}^{2}$, at output of the receiver is calculated as

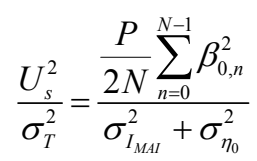

where $\sigma_{I_{M A I}}^{2}$ is the variance of $I_{M A I}$, which is shown in (14). In the limiting case of large $N$ and by the methods of CLT (central limit theory), the MAI can be approximated by a Gaussian r.v. with zero mean and the variance, $\sigma_{I_{M A I}}^{2}$, can be determined as

$$
\begin{aligned}
\sigma_{I_{M A I}}^{2}= & E\left[I_{M A I}^{2}\right]=\frac{P}{2}(k-1) \cdot E\left[\beta_{k, n}^{2}\right] \\
& \cdot E\left[\cos ^{2} \bar{\theta}_{k, n}\right]=\frac{P}{4}(k-1) \Omega_{k, n}
\end{aligned}
$$

where $\Omega_{k, n}=E\left[\beta_{k, n}^{2}\right], E\left[\cos ^{2} \bar{\theta}_{k, n}\right]=1 / 2$. On the other hand, the background noise term $\eta_{0}$ is a random variable with zero mean and the variance can be calculated as

$$
\sigma_{\eta_{0}}^{2}=E\left[\eta_{0}^{2}\right]=\frac{N N_{0}}{4 T_{b}}
$$

By substituting (14) and (15) into (13), which can be obtained as

$$
\frac{U_{s}^{2}}{\sigma_{T}^{2}}=\frac{1}{2 N} \cdot S_{N}\left(\sigma_{0}\right)^{-1}
$$

where

$$
S_{N}=\sum_{n=0}^{N-1} \beta_{0, n}^{2} / \Omega_{k, n}
$$

and

$$
\sigma_{0}=\frac{N N_{0}}{4 P T_{b} \Omega_{k, n}}+\frac{k-1}{4}=\frac{N}{4 \gamma_{0}}+\frac{k-1}{4},
$$

where

$$
\gamma_{0}=P T_{b} \Omega_{k, n} / N_{0}=E_{b} \Omega_{k, n} / N_{0}
$$

is the $S N R$ of each bit, and $E_{b}=P T_{b}$ denotes the bit energy.

It is known that the decision variable in (13) has a Gaussian distribution conditioned on the uncorrelated and correlated channel power $\beta_{0, n}^{2}$, respectively, and the AWGN, $\eta_{0}$, and the MAI, $\eta_{M A I}$ are mutually independent. Therefore, the probability of error by means of BPSK modulation conditioned on the instantaneously SNR has been given in (16) can be evaluated as follows.

If the conditions of correlated channels are considered as the impact factors for an MC-CDMA system, then the average bit error probability for the case can be calculated by averaging (5) and (16), and yield as

$$
\begin{aligned}
\boldsymbol{P}_{e}= & \frac{1}{2 \pi} \int_{0}^{\pi / 2} \int_{0}^{\infty} \exp \left(-\frac{2 x \cdot \sin ^{2} \theta}{\frac{(K-1) d(L, \delta)}{N^{2}} \Omega_{0}+\frac{N_{0}}{E_{b}}}\right) \\
& \sum_{l=1}^{L} \sum_{\gamma=1}^{m} \beta_{l_{\gamma}} p_{\gamma}(x, l, \gamma) d x d \theta
\end{aligned}
$$

Next, by using of the integral equivalent formula,

$$
\int_{0}^{\infty} e^{-\mu \gamma} \gamma^{m-1} d \gamma=1 / \mu^{m} \Gamma(m)
$$

the average BER can be simplified and expressed as

$$
\begin{aligned}
P_{e} & =\frac{1}{2} \int_{0}^{\infty} Q\left(\sqrt{\frac{2 S_{N}}{\frac{(K-1) d(L, \delta)}{N^{2}} \Omega_{0}^{\prime}+\frac{N_{0}}{E_{b}}}}\right) f_{\gamma}(x) d x \\
& =\frac{1}{2 \pi} \sum_{l=1}^{L} \sum_{\gamma=1}^{m} \beta_{l_{\gamma}}\left(\frac{1}{\lambda_{l}}\right)^{\gamma} \\
& \int_{0}^{\pi / 2}\left[\frac{2}{\left(\frac{(K-1) d(L, \delta)}{N^{2}} \Omega_{0}+\frac{N_{0}}{E_{b}}\right) \sin ^{2} \theta}+\frac{1}{\lambda_{l}}\right]^{-\gamma} d \theta
\end{aligned}
$$


where $\lambda_{\ell}$ is given in (7), $\lambda_{1}$ and $\sigma_{0}$ are shown in (6) and (22), respectively, the symbol ${ }_{2} F_{1}(\cdot, \cdot ; \cdot \cdot)$ denotes the confluent hyper-geometric function [13], and $\Omega_{f}=[d(L, \xi) / N] \Omega_{0}$, which represent that the exponential MIP (multipath intensity profile) is adopted in this derivation, and the $d(L, \xi)=1-e^{-L \xi} / 1-e^{-\xi}$.

\section{Results and Discussions}

In this section where some of the numerical results for validation of the derived formulas are discussed, for the reason of the impact in the different configuration of antenna array, it is interesting to review the view point of space diversity (antenna diversity) first, the numerical results will be followed up.

\subsection{Discussion of Space Diversity}

It is well known that adopts the diversity scheme is one common mean to overcome the degradation in performance of a wireless communication system due to fading (generally small fading). Generally speaking, the diversity reception may be accomplished by a number of techniques. The most common approaches, employed in wireless radio applications, are frequency diversity, time diversity, multipath diversity, path diversity, and antenna diversity. In antenna diversity, also called space diversity, which is divided into classes such as, field component, space, polarization, and angle [14]. Accordingly, the different results from the phenomenon of channel fading will be solved by a lot of different methods. For a multiple-access spread-spectrum wireless system, e.g., an

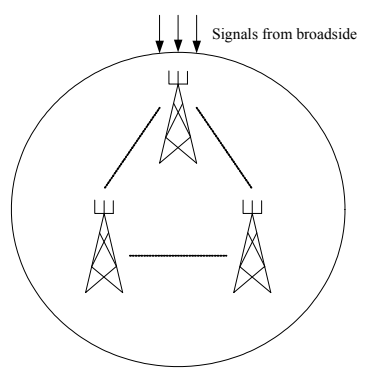

(a) Triangular geometric

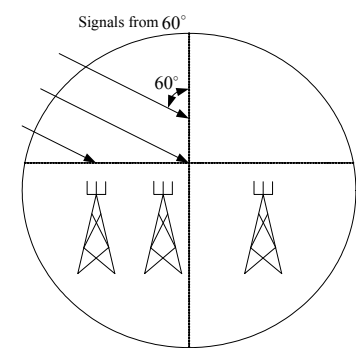

(b) Linear geometric

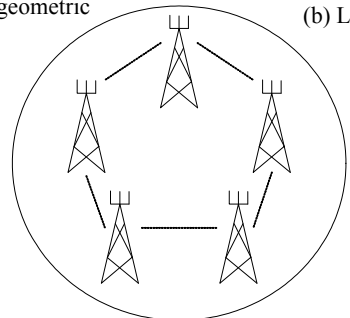

(c) Circular geometric

Figure 1. Three different antenna array geometrics applied in this paper.

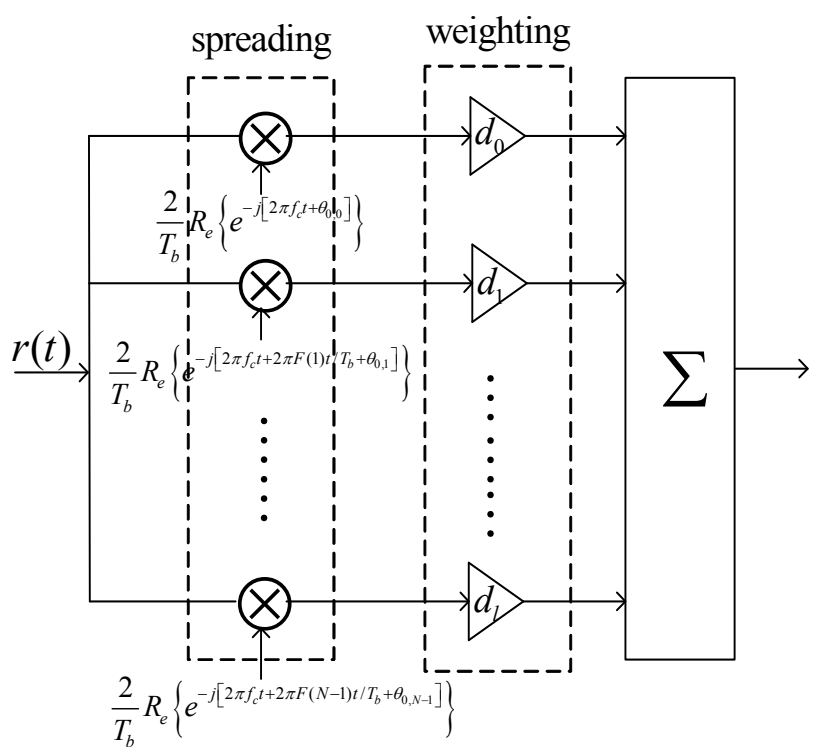

Figure 2. The receiver model of an MC-CDMA system.

MC-CDMA system, when it is working in the environment suffers from the frequency selective fading, the space diversity is a pretty one mean which is able to be for utilizing to combine with different antenna array in order to overcome the fading problems. In this article, three kinds of antenna constructions, uniform linear with 3 -fixed antennas, triangular array with 3 -fixed antennas, and circular array with 4-fixed antennas, as applied and depicted in Figure 1, will be co-existed with the receiver, which is shown in Figure 2, of an MC-CDMA system. The effects of correlation generated between different antennas will be taken into account the analysis. Therefore, the depth of correlation will be characterized by eigenvalues, which are calculated from the covariance matrix formed by a given Gaussian branch (antenna) correlation model as [5]

$$
C_{i, j}(d / \lambda)=\exp \left[-k(i-j)^{2}(d / \lambda)^{2} / 2\right]
$$

where $i, j=1, \cdots, L, d$ denotes the physical distance of two adjacent antennas, and $\lambda$ is the wavelength of incoming waveform. It means that the ratio $d / \lambda$ is going to be a factor dominates the performance of a wireless radio system while considering the effect of antenna (branch) correlation. The curves with varying $d / \lambda$ values are shown in Figure 3. Hereafter, the spatial correlation for a circular antenna array geometric investigated in [15] is adopted to verify the accuracy of the derived formulas. Since the Gaussian correlation model is implied in the cases of linear and triangular antenna geometrics mentioned previously, one of the correlation function, $\rho(i, j)$, determined in [15] with the Gaussian angle distribution for the incident signal is applied and rewritten as 


$$
\begin{gathered}
\operatorname{Re}[\rho(i, j)]=\frac{k}{\sqrt{\pi}} \int_{-\frac{\pi}{\sqrt{2} \rho}}^{\frac{\pi}{\sqrt{2} \rho}} e^{-\gamma^{2}} \\
{\left[J_{0}\left(Z_{C}\right)+2 \sum_{k=1}^{\infty} J_{2 k}\left(Z_{C}\right)\right.} \\
\cos (2 k(\gamma+\sqrt{2} \sigma \gamma+\phi))] d y \\
\operatorname{Im}[\rho(m, n)]=\frac{k}{\sqrt{\pi}} \int^{\frac{\pi}{\sqrt{2} \sigma}} e^{-\gamma^{2}} \\
{\left[2 \sum_{k=0}^{\infty} J_{2 k+1}\left(Z_{C}\right) \cdot \sin ((2 k+1)\right.} \\
(\gamma+\sqrt{2} \sigma \gamma+\phi))] d y
\end{gathered}
$$

where $\operatorname{Re}[\cdot]$ and $\operatorname{Im}[\cdot]$ denote the real part and imaginary part, respectively, $\kappa=1 / \operatorname{erf}\left(\frac{\pi}{\sqrt{2} \sigma}\right)$, where $\operatorname{erf}(\cdot)$ is the error function, $\sigma$ indicates the standard deviation of the Gaussian distribution, $J_{n}(\cdot)$ is the modified Bessel function of the first kind, $Z_{C}=\left(Z_{1}^{2}+Z_{2}^{2}\right)^{0.5} \quad, \quad$ where $\quad Z_{l}=2 \pi|i-j|(d / \lambda)$, $i, j=1, \ldots, L, \phi$ represents the mean angle of arrival.

Hence, for a given antenna array configuration, the branch correlation depends on the signal incident angle, the antenna height, and the antenna separation. These effects for the system performance will be figured out in sub-section.

\subsection{Numerical Results}

In order to validate the accuracy of our derived formulas

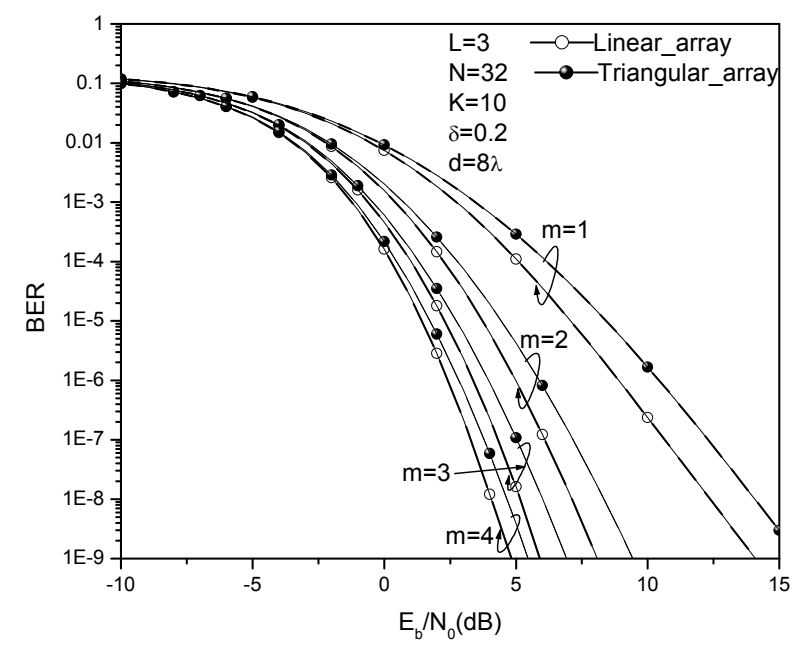

Figure 3 BER vs SNR with different $m$ values between linear and triangular array. mentioned above and the comparison purpose, the system performance for both linear and triangular antenna array geometrics are shown in Figure 3 together. It is worthy noting that the system performance always become degraded when the system is operating in the triangular antenna array. It is well known that the system performance is significantly varied with different separations between transmitter and receiver. The system performance for an MC-CDMA system under the circular antenna array with different fading parameter, $m=2$ is presented in Figure 4. The incident angle of the signal with $\theta=0^{\circ}$, and $45^{\circ}$ are assumed in both figures. It is valuable to mention that the system will stay superior situation when the incident is in small one, this is due to the correlation will become decrease after the incident angle of the propagating waveform related small.

\section{Conclusions}

In this paper the impact of distinctive structure of antenna with branch correlation for an MC-CDMA system, operating over the frequency selective fading environments is studied. The system parameters with the subcarrier number, correlation coefficients, the branch number, and the exponential MIP are considered for determination the system performance of an MC-CDMA system. The results explicit illustrated that the phenomena of channel correlation and the multipath fading do dominate the performance of MC-CDMA communication systems. However, the most important factors should be the fading parameter of the fading model, and the subcarrier number. Hence it is worthy not only to pay much attention in the consideration of correlation coefficient for channel fading while designing the MC-CDMA systems, but the chosen of designing the scenarios of antenna are important.

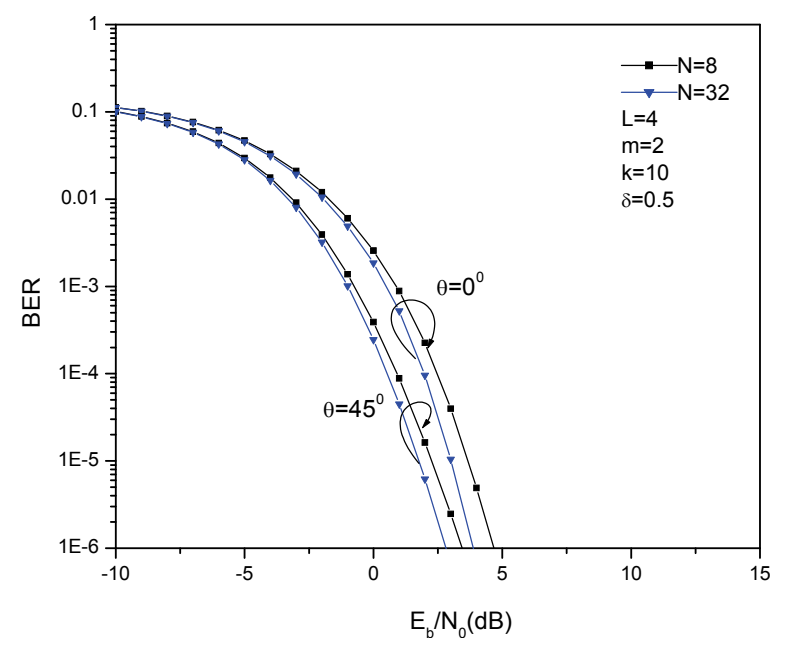

Figure 4. Plots BER vs SNR with different subcarrier number under circular antenna array, and the fading parameter is $m=2$. 


\section{References}

[1] N. Yee, J. P. Linnartz, and G. Fettweis, "Multi-carrier CDMA in indoor wireless radio networks," IEICE Transactions on Communication, Vol. E77-B, No. 7, pp. 900-904, July 1994.

[2] Z. J. Kang and K. Yao, "Performance comparison of MC-CDMA over frequency-selective Nakagami- $m$ and Rayleigh fading channels," Vehicular Technology Conference, Vol. 6, pp. 4228-4232, September 2004.

[3] E. A. Sourour and M. Nakagami, "Performance of orthogonal multicarrier CDMA in a multipath fading channel," IEEE Transactions on Communication, Vol. 44, pp. 356-367, March 1996.

[4] T. Kim, Y. Kim, J. Park, K. Ko, S. Choi, C. Kang, and D. Hong, "Performance of an MC-CDMA system with frequency offsets in correlated fading," IEEE International Conference on ICC 2000, Vol. 2, pp. 1095-1099, June 2000.

[5] J. Park, J. Kim, S. Choi, N. Cho, and D. Hong, "Performance of MC-CDMA systems in non-independent Rayleigh fading," IEEE on ICC' 99, Vol. 1, pp. 6-10, 506-510, June 1999.

[6] Q. R. Shi and M. Latva-aho, "Exact error floor for downlink MC-CDMA with maximal ratio combining in correlated Nakagami fading channels," 2002 International Zurich Seminar on Broadband Communications, pp. 37-1-37-5, February 20, 2002.

[7] Q. Shi and M. Latva-aho, "Performance analysis of MC-CDMA in Rayleigh fading channels with correlated envelopes and phases," IEE Proceedings: Communication, Vol. 150, No. 3, pp. 214-220, June 2003.
[8] Z. Li and M. Latva-aho, "Erroe probability for MC-CDMA in Nakagami-m fading channels using equal gain combining," IEEE International Conference on ICC 2002, Vol. 1, pp. 227-231, April 28-May 2, 2002.

[9] M. S. Alouini, A. Abdi, and M. Kaveth, "Sum of gamma variates and performance of wireless communication systems over Nakagami- $m$ fading channels," IEEE Transactions on Vehicular Technology, Vol. 50, No. 6, pp. 1471-1480, November 2001.

[10] M.Nakagami, "The m-distribution-A general formula of intensity distribution of rapid fading," Statistic Methods of in Radio Wave Propagation, Pergamon Press, New York, pp. 3-36, 1960.

[11] M. Schwartz, W. R. Bennett, and S. Stein, "Communication systems and techniques," McGraw-Hill, New York, 1966.

[12] M. K. Simon and M. S. Alouini, "A unified approach to the performance analysis of digital communication over generalized fading channel," Proceedings of the IEEE, Vol. 86, pp. 1860-1877, September 1998.

[13] I. S. Gradshteyn and I. M. Ryzhik. "Table of integrals, series, and products," CA: Academic Press, San Diego, 5th Edition, 1994.

[14] W. C. Jakes, "Microwave Mobile Communications," IEEE Press, Piscataway, Jersey, 1974.

[15] J. Zhou, S. Sasaki, S. Muramatsu, H. Kikuchi and Y. Onozato, "Saptial correlation for a circular antenna array and its applications in wireless communications," IEEE Global Telcommunications Conference, Vol. 2, pp. 1108-1113, 2003. 